\title{
Analysis of Decentralized Estimation Filters for Formation Flying Spacecraft
}

\author{
Milan Mandic,, Louis Breger $†$ and Jonathan P. How ${ }^{\ddagger}$ \\ MIT Department of Aeronautics and Astronautics
}

\begin{abstract}
Future formation flying missions are being planned for fleets of spacecraft in MEO, GEO, and beyond where relative navigation using GPS will either be impossible or insufficient. To perform fleet estimation for these scenarios, local ranging devices on each vehicle are being considered to replace or augment the available GPS measurements. A previous paper presented several approaches for distributing the computational load of the estimation process across the vehicles in the fleet. This paper extends the previous work to present a more detailed investigation of the covariances for the different distributed estimation algorithms. This enables an analysis of the transient effects as the ranging measurements are added to the estimator, which extends the previous steady-state comparison. This transient covariance analysis more clearly shows the effect of ignoring errors in the ranging measurements that result when the location of the target vehicle is also not well known. These results provide further insight on the relative performance of these filters and identifies the Schmidt covariance correction (SCC), the essence of the Schmidt Kalman Filter, as being responsible for better performance of Schmidt Kalman Filter compared to other decentralized filters.
\end{abstract}

\section{Introduction}

$\mathrm{T}$ HE concept of formation flying of satellite clusters has been identified as an enabling technology for many types of space science missions. 1 It has also been identified as one of seventeen enabling technologies for the new NASA exploration initiative. ${ }^{2}$ An example of this is ground exploration of remote destinations, where having a group of vehicles working together may be far more efficient than a single vehicle. The use of fleets of smaller vehicles instead of one monolithic vehicle should (i) improve the science return through longer baseline observations, (ii) enable faster ground track repeats, and (iii) provide a high degree of redundancy and reconfigurability in the event of a single vehicle failure. The GN\&C tasks are very complicated for larger fleets because of the size of the associated estimation and control problems and the large volumes of measurement data available. As a result, distributing the guidance and control algorithms becomes a necessity in order to balance the computational load across the fleet and to manage the inter-spacecraft communication. This is true not only for the optimal planning, coordination, and control ${ }^{[3}$ but also for the fleet state estimation, since the raw measurement data is typically collected in a decentralized manner (i.e., each vehicle takes its own local measurements).

GPS can be used as an effective sensor for many space applications, but it requires constant visibility of the GPS constellation. In space, GPS visibility begins to breakdown at high orbital altitudes (e.g. highly elliptic, GEO, or at L2). Thus, a measurement augmentation is desired to permit relative navigation through periods of poor visibility and also to improve the accuracy when the GPS constellation is visible $5[6] 7[8] 9$ However, the local range measurements taken onboard the spacecraft strongly correlate the states of the vehicles, which destroys the block-diagonal nature of the fleet measurement matrix ${ }^{4}[0$ and greatly complicates the process of decentralizing the algorithms. ${ }^{[12}$ In contrast to the GPS-only estimation scenario, which effectively decentralizes for reasonable fleet separations, this estimation problem does not decorrelate at any level. As a result, Ref. 12 investigated several methods to efficiently decentralize the estimation algorithms while retaining as much accuracy as possible.

*Research Assistant, MIT Department of Aeronautics and Astronautics, mandicm@mit.edu

${ }^{\dagger}$ Research Assistant, MIT Department of Aeronautics and Astronautics, lbreger@mit.edu

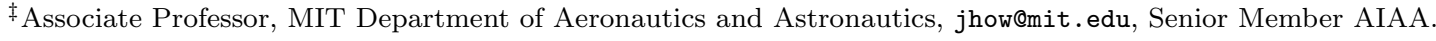


To populate the decentralized architectures, Ref. 12 developed a new approach to estimation based on the Schmidt Kalman Filter (SKF). The SKF was shown to work well as a reduced-order decentralized estimator because it correctly accounts for the uncertainty present in the local ranging measurements, which is a product of not knowing the location of the other vehicles in the fleet. Since this correction is applied to the covariance of the measurement, this property of the SKF is called the Schmidt covariance correction (SCC). We extend the covariance comparison in Ref. 12 to consider the transients that occur as ranging measurements are added to the estimator. Previous work performed a similar comparison on the steadystate covariances from the various filter and architecture options. This enabled a comparison of the filter performances, but it could not explain why some of the decentralized techniques performed better than others. The investigation in this paper of what we call the "transient response" of the filter provides further insight on the relative performance of these filters. This analysis also indicates the advantage of using the SCC, which can be extended to other estimation algorithm/architectures. The following section discusses prior work on reduced-order decentralized filters, which is followed by a detailed investigation of the covariance for different algorithms.

\section{Reduced-order Decentralized Filters}

Recent work by Park ${ }^{13}$ introduced the Iterative Cascade Extended Kalman Filter (ICEKF), a reducedorder estimation algorithm for use in decentralized architectures. This filter is used for local ranging augmentation in applications where GPS-only measurements are not sufficient. The ICEKF filter uses an iterative technique that relies on communication between each vehicle in the fleet and continues until a specified level of convergence is reached. It was shown that the ICEKF can incorporate local ranging measurements with GPS levels of accuracy, producing nearly optimal performance. However, Ref. 14 demonstrated that the filter performance can deteriorate when highly accurate local measurements (i.e., more accurate than GPS) are added, and that this performance loss occurs when error/uncertainty in the relative state vectors is not correctly accounted for in the filter.

One way to account for this uncertainty in the relative state is to include it in the measurement noise covariance $R$, which is the approach taken in the Bump Up $R$ method:

$$
R_{\text {bump }}=R+J P_{\text {yу }} J^{T}
$$

where $J$ is the measurement matrix for all non-local measurements in the fleet and $P_{y y}$ is the initial covariance matrix for all non-local states in the fleet state vector. Equation 1 implies that the measurements now have larger noise covariance, making them less accurate than was initially assumed.

Another approach examined in Ref. 14 is the Schmidt Kalman Filter (SKF). This filter also increases the variances in the $R$ matrix, but in contrast to Bump $U p R$, this approach is dynamic and also accounts for the off-diagonal blocks of the error covariance. The SKF eliminates non-local state information, thereby reducing the computational load on the processor. This elimination is accomplished by partitioning the measurement and propagation equations:

$$
\begin{gathered}
{\left[\begin{array}{l}
x \\
y
\end{array}\right]_{k+1}=\left[\begin{array}{cc}
\phi_{x} & 0 \\
0 & \phi_{y}
\end{array}\right]_{k}\left[\begin{array}{l}
x \\
y
\end{array}\right]_{k}+\left[\begin{array}{l}
w_{x} \\
w_{y}
\end{array}\right]_{k}} \\
z_{k}=\left[\begin{array}{ll}
H & J
\end{array}\right]_{k}\left[\begin{array}{l}
x \\
y
\end{array}\right]_{k}+\nu_{k} \\
P_{k}=\left[\begin{array}{ll}
P_{x x} & P_{y x} \\
P_{x y} & P_{y y}
\end{array}\right]_{k}
\end{gathered}
$$

where $x$ represents the vector containing the states of interest (called the local state, which includes the positions, velocities, and time of the vehicle relative to the fleet origin) and $y$ represents the remaining states (i.e., the positions, velocities, and time of all other vehicles relative to the fleet origin). After partitions of Eqs. 2 and 3 are applied to the general Kalman filter equations, each block is solved, and the gain for the $y$ states is set to zero! 15

$$
\alpha_{k}=H_{k} P_{x x_{k}}^{-} H_{k}^{T}+H_{k} P_{x y_{k}}^{-} J_{k}^{T}+
$$




$$
\begin{aligned}
& J_{k} P_{y x_{k}}^{-} H_{k}^{T}+J_{k} P_{y y_{k}}^{-} J_{k}^{T}+R_{k} \\
K_{k}= & \left(P_{x x_{k}}^{-} H_{k}^{T}+P_{x y_{k}}^{-} J^{T}\right) \alpha_{k}^{-1} \\
\hat{x}_{k}^{+}= & \hat{x}_{k}^{-}+K_{k}\left(z_{k}-H_{k} \hat{x}_{k}^{-}-J_{k} \hat{y}_{0}\right) \\
P_{x x_{k}}^{+}= & \left(I-K_{k} H_{k}\right) P_{x x_{k}}^{-}-K_{k} J_{k} P_{y x_{k}}^{-} \\
P_{x y_{k}}^{+}= & \left(I-K_{k} H_{k}\right) P_{x y_{k}}^{-}-K_{k} J_{k} P_{y y_{k}}^{-} \\
P_{y x_{k}}^{+}= & P_{x y_{k+1}}^{-T} \\
P_{y y_{k}}^{+}= & P_{y y_{k}}^{-}
\end{aligned}
$$

Schmidt-Kalman Time Update

$$
\begin{aligned}
\hat{x}_{k+1}^{-} & =\phi_{x_{k}} \hat{x}_{k}^{+} \\
P_{x x_{k+1}}^{-} & =\phi_{x_{k}} P_{x x_{k}}^{+} \phi_{x_{k}}^{T}+Q_{x_{k}} \\
P_{x y_{k+1}}^{-} & =\phi_{x_{k}} P_{x y_{k}}^{+} \phi_{y_{k}}^{T} \\
P_{y x_{k+1}}^{-} & =P_{x y_{k+1}}^{-T} \\
P_{y y_{k+1}}^{-} & =\phi_{y_{k}} P_{y y_{k}}^{+} \phi_{y_{k}}^{T}+Q_{y_{k}}
\end{aligned}
$$

In order for the SKF to compute an appropriate amount to increase $R$, each spacecraft communicates both its local state vector and its local error covariance matrix to the next spacecraft in the fleet. This change to $R$ is called the Schmidt Covariance Correction. The additional error covariance information, which is not transmitted when using Bump $U p R$, allows a more appropriate correction, but also requires additional inter-spacecraft communication.

The analysis performed in Ref. 14 showed that the error covariance in the ICEKF method is relatively close to the error covariance of the optimal, centralized case. However, this observation was described as misleading, because it was not a good indicator of the filter's performance. The filter's unrealistically high trust in the measurements, due to the assumed low error covariance, was conjectured to be the primary reason for this poor performance. The following section explores this point in more detail. It also presents an equivalent derivation for the SKF approach, which demonstrates how increasing the measurement noise covariance improves the actual filter performance.

\section{Covariance Comparison}

Previous research in Refs. 11,12 and 13 showed that results from the ICEKF are worse than might be expected. The ICEKF produces these poor results, because it makes unrealistic assumptions about the uncertainty associated with the ranging measurements from the other vehicles, since this filter does not include the position uncertainty of other vehicles. These unrealistic assumptions are captured in the measurement noise covariance $(R)$, which provides a measure of the "quality" of each measurement. One way to investigate this problem is to analyze the error covariance matrix $(P)$, and to understand the true impact we take the approach of investigating the transients immediately after ranging measurements are added to the estimator. This approach differs from that of Ref. [12, which compares steady-state covariances. The steady state values are a good way to compare the overall performance values, but they tend to obscure the reasons why some filters diverge and others do not. Using the transient covariance analysis more clearly shows the difference in covariances that occur when new, corrupt measurements are introduced to the filter, as is shown in Figure 1. This figure was obtained for a SISO system, for which Bump Up R is essentially equivalent to the Schmidt-Kalman Filter (SKF). Analyzing $P$ across the transient step should show how the incorrect modeling of $R$ impacts the filter's confidence in the state estimates. The ICEKF filter error covariance is much lower than it should be at this stage of the estimation, thereby corrupting all future measurement updates. The analytic derivation of this phenomenon is provided in Section A. The results also show that this problem can be partially alleviated by increasing the $R$ value in the algorithm using a systematic approach, such as the Bump Up R or SKF formulations. For the scalar case, the error covariances resulting from the two methods are related by:

$$
P_{\mathrm{bu}}^{+}>P_{\mathrm{f}}^{+}
$$




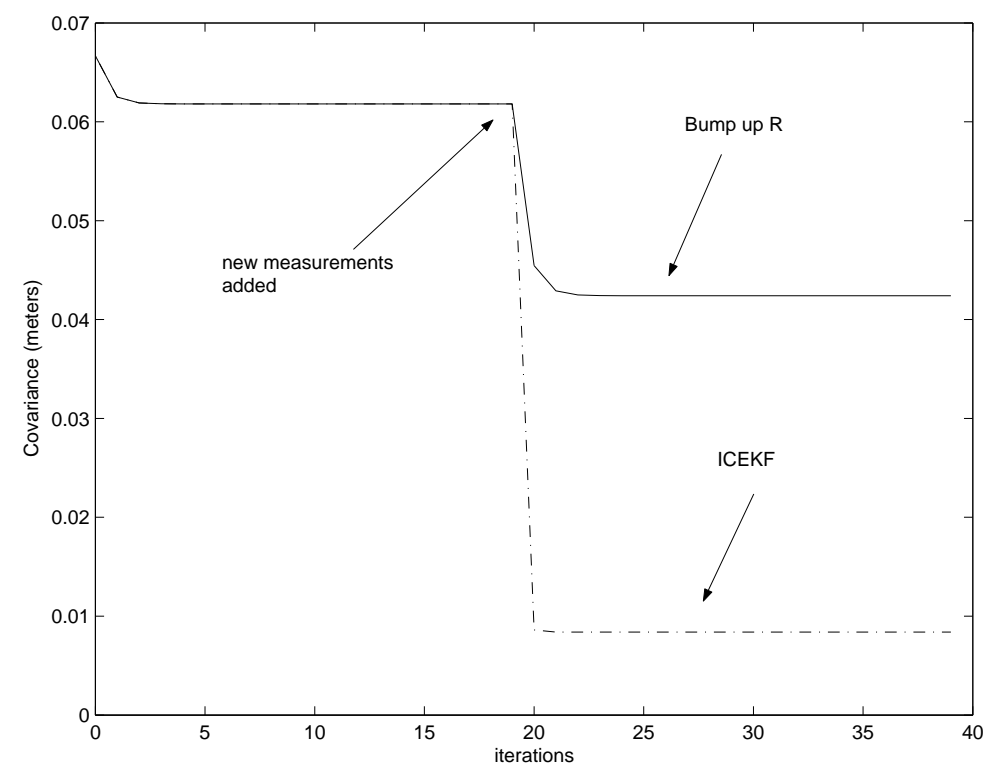

Figure 1. ICEKF and Bump-Up R; at the 20th iteration, new measurements are introduced

where the error covariance matrix $P_{\mathrm{f}}$ is analyzed using $R_{\mathrm{f}}$ with the gain based on $R_{\mathrm{f}}$, and $P_{\mathrm{bu}}$ is analyzed using $R_{\mathrm{bu}}$ with gain based on $R_{\mathrm{bu}}$. After the update step, the error covariance using the Bump Up $R$ approach $\left(P_{\mathrm{bu}}^{+}\right)$is larger than the ICEKF result $\left(P_{\mathrm{f}}^{+}\right)$, so Bump Up $R$ should avoid the problems with the ICEKF technique.

Since these analytic results were based on several approximations, a simulation was conducted to confirm that the Bump Up $R$ method performed better than ICEKF regardless of the assumptions. This simulation computed the transient behavior of the error covariance using various filters that were based on different assumptions about the measurement noise covariances $R$. The results are shown in Figures 2 and 4 . These results show that the Bump $U p R$ method provides a better prediction of the best possible filter performance, confirming the analytical predictions. Similar results would be expected for the SKF, due to its equivalence with the Bump Up $R$ method in a scalar case.

\section{A. Effects Of Using Corrupted Measurements}

The purpose of this section is to analyze the impact of adding new ranging measurements to the estimator, and in particular, to determine how the covariance matrix changes. The bumped-up case is included, because our ultimate goal is to show the effect of using the SCC on incorporating new measurements in a filter. The Schmidt-Kalman filter is a dynamic version of the Bump Up $R$ filter, in the sense that it uses a better technique to increase the noise covariance. First, the analysis will be restricted to the scalar case, which should provide adequate insights into the result. Second, the values associated with the actual values are noted with the subscript $a$, filter values with the subscript $f$, and bumped-up values with the subscript $b u$. In the following derivation, $R_{\mathrm{a}}>R_{\mathrm{bu}}>R_{\mathrm{f}}$. The derivation begins with a measurement update for the error covariance $^{15}$

$$
P^{+}=(I-K H) P^{-}(I-K H)^{T}+K R K^{T}
$$

where $K$ is the Kalman gain, $I$ is an identity matrix, and $H$ is the measurement matrix. The symbols $I$ and $H$ represent scalars in this case. The error covariance $P^{-}$represents the filter error covariance based on the measurements available prior to adding the set of ranging measurements. These measurements could be obtained from GPS or other external sources. Since the new, added ranging measurements are more accurate than the previous, the error covariance should substantially decrease. It is essential to observe the transient behavior of the covariance in the first step after the new measurements are added, because the filter performance is heavily impacted at that time. For example, if the measurement noise $(R)$ is too low, the filter might continue to have a high confidence in erroneous measurements and the estimation results 


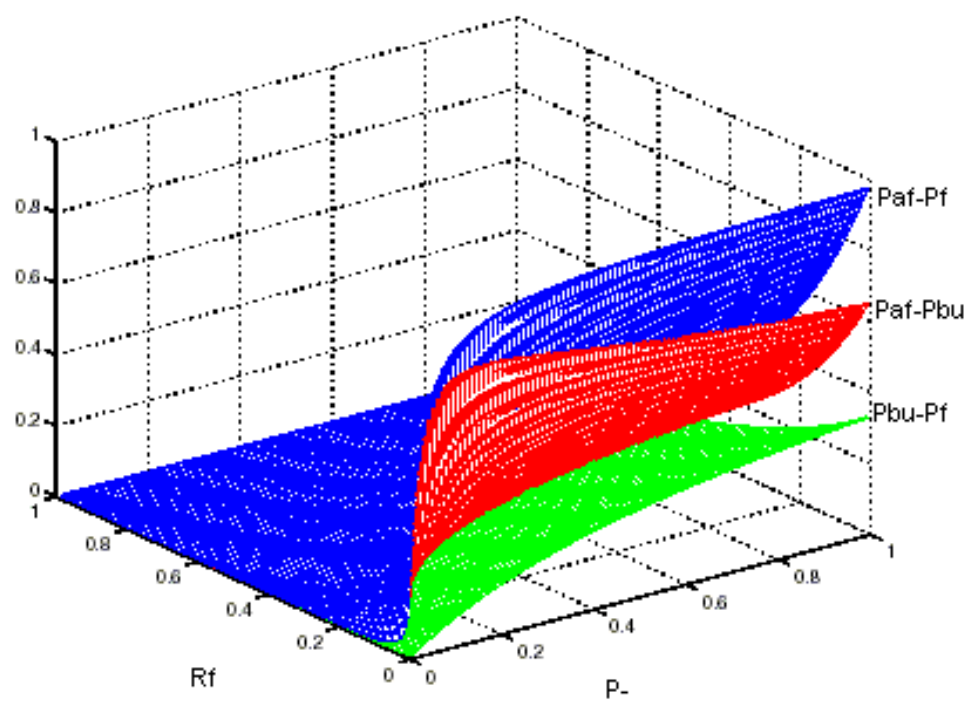

Figure 2. Error Covariance Comparison. Differences between the various covariances are all non-negative, which means that $P_{\mathrm{af}}^{+}>P_{\mathrm{bu}}^{+}>P_{\mathrm{f}}^{+}$.

will degrade. In the scalar case, Eq. 18 can be rewritten as

$$
P^{+}=(I-K H)^{2} P^{-}+K^{2} R
$$

The corresponding Kalman gain is

$$
K=P^{-} H^{T}\left(H P^{-} H^{T}+R\right)^{-1}=\frac{P^{-} H}{H^{2} P^{-}+R}
$$

Thus, for a filter using the incorrect covariance $R=R_{\mathrm{f}}$, the gain matrix would be

$$
K=\frac{P^{-} H}{H^{2} P^{-}+R_{\mathrm{f}}}
$$

in which case Eq. 19 can be rewritten as

$$
P_{\mathrm{af}}^{+}=\left(1-\frac{P^{-} H^{2}}{H^{2} P^{-}+R_{\mathrm{f}}}\right)^{2} P^{-}+\left(\frac{P^{-} H}{H^{2} P^{-}+R_{\mathrm{f}}}\right)^{2} R_{\mathrm{a}}
$$

where $P_{\mathrm{af}}$ is used to designate that this corresponds to the "actual" error covariance that one might expect when using this filter. Essentially the gain is based on the assumed $R_{\mathrm{f}}$, but the error analysis is based on the more realistic $R_{\mathrm{a}}$. To proceed, two quantities are defined

$$
\begin{aligned}
M & \equiv\left(1-\frac{P^{-} H^{2}}{H^{2} P^{-}+R_{\mathrm{f}}}\right)^{2} P^{-} \\
N & \equiv\left(\frac{P^{-} H}{H^{2} P^{-}+R_{\mathrm{f}}}\right)^{2}
\end{aligned}
$$

So that Eq. 22 becomes

$$
P_{\mathrm{af}}^{+}=M+N R_{\mathrm{a}}
$$

and similarly

so, from Eqs. 25 and 26

$$
P_{\mathrm{f}}^{+}=M+N R_{\mathrm{f}}
$$

$$
P_{\mathrm{af}}^{+}-P_{\mathrm{f}}^{+}=N\left(R_{\mathrm{a}}-R_{\mathrm{f}}\right)>0 \quad \Rightarrow \quad P_{\mathrm{af}}^{+}>P_{\mathrm{f}}^{+}
$$




\section{Comparing $P_{\mathrm{abu}}$ and $P_{\mathrm{bu}}$ With $P_{\mathrm{af}}$ and $P_{\mathrm{f}}$}

The second step in this derivation is to compare $P_{\mathrm{abu}}$ and $P_{\mathrm{bu}}$ with $P_{\mathrm{af}}$ and $P_{\mathrm{f}}$. Consider the case where a modified value of the measurement covariance $R_{\mathrm{bu}}$ is developed using the Bump Up $R$ algorithm. Starting from the equations for the error covariance in Eq. 22

$$
\begin{aligned}
P_{\mathrm{af}}^{+} & =\left(1-\frac{P^{-} H^{2}}{H^{2} P^{-}+R_{\mathrm{f}}}\right)^{2} P^{-}+\left(\frac{P^{-} H}{H^{2} P^{-}+R_{\mathrm{f}}}\right)^{2} R_{\mathrm{a}} \\
P_{\mathrm{bu}}^{+} & =\left(1-\frac{P^{-} H^{2}}{H^{2} P^{-}+R_{\mathrm{bu}}}\right)^{2} P^{-}+\left(\frac{P^{-} H}{H^{2} P^{-}+R_{\mathrm{bu}}}\right)^{2} R_{\mathrm{bu}}
\end{aligned}
$$

several assumptions and approximations are made to compare these error covariances. For the scalar case, define $\gamma=P^{-} H^{2}$, then

$$
\begin{aligned}
& P_{\mathrm{af}}^{+}=\left(1-\frac{\gamma}{\gamma+R_{\mathrm{f}}}\right)^{2} P^{-}+\left(\frac{\gamma}{\gamma+R_{\mathrm{f}}}\right)^{2} \frac{R_{\mathrm{a}}}{H^{2}} \\
& P_{\mathrm{bu}}^{+}=\left(1-\frac{\gamma}{\gamma+R_{\mathrm{bu}}}\right)^{2} P^{-}+\left(\frac{\gamma}{\gamma+R_{\mathrm{bu}}}\right)^{2} \frac{R_{\mathrm{bu}}}{H^{2}}
\end{aligned}
$$

Now if $R_{\mathrm{f}} \ll \gamma$, which is equivalent to assuming that the new measurements are much more accurate than the previous, then the following is true

$$
\frac{y}{y+x} \approx 1-\frac{x}{y}, \quad x \ll y
$$

Then Eq. 28 reduces as follows

$$
\begin{aligned}
P_{\mathrm{af}}^{+} & \approx\left(1-\left(1-\frac{R_{\mathrm{f}}}{\gamma}\right)\right)^{2} P^{-}+\left(1-\frac{R_{\mathrm{f}}}{\gamma}\right)^{2} \frac{R_{\mathrm{a}}}{H^{2}} ; \quad \frac{R_{\mathrm{f}}}{\gamma} \ll 1 \\
& =\left(\frac{R_{\mathrm{f}}}{\gamma}\right)^{2} P^{-}+\left(1-\frac{R_{\mathrm{f}}}{\gamma}\right)^{2} \frac{R_{\mathrm{a}}}{H^{2}} \approx \frac{R_{\mathrm{a}}}{H^{2}}
\end{aligned}
$$

The error covariance for the bumped up method, Eq. 29 becomes

$$
\begin{aligned}
P_{\mathrm{bu}}^{+} & =\left(\frac{\gamma+R_{\mathrm{bu}}-\gamma}{\gamma+R_{\mathrm{bu}}}\right)^{2} P^{-}+\left(\frac{\gamma}{\gamma+R_{\mathrm{bu}}}\right)^{2} \frac{R_{\mathrm{bu}}}{H^{2}} \\
& =\frac{R_{\mathrm{bu}}^{2} P^{-}}{\left(\gamma+R_{\mathrm{bu}}\right)^{2}}+\frac{\gamma^{2} R_{\mathrm{bu}}}{H^{2}\left(\gamma+R_{\mathrm{bu}}\right)^{2}} \\
& =\frac{R_{\mathrm{bu}}^{2} \gamma+\gamma^{2} R_{\mathrm{bu}}}{H^{2}\left(\gamma+R_{\mathrm{bu}}\right)^{2}}=\frac{R_{\mathrm{bu}} \gamma\left(\gamma+R_{\mathrm{bu}}\right)}{H^{2}\left(\gamma+R_{\mathrm{bu}}\right)^{2}}=\frac{R_{\mathrm{bu}} \gamma}{H^{2}\left(\gamma+R_{\mathrm{bu}}\right)} \\
& =\frac{R_{\mathrm{bu}} P^{-}}{\gamma+R_{\mathrm{bu}}}
\end{aligned}
$$

With these results, a comparison can be drawn between $P_{\text {af }}$ and $P_{\mathrm{bu}}$. Assuming

$$
P_{\mathrm{af}}^{+}-P_{\mathrm{bu}}^{+}>0
$$

which, using Eqs. 34 and 38 , can be rewritten as

$$
\begin{aligned}
\frac{R_{\mathrm{a}}}{H^{2}}>\frac{R_{\mathrm{bu}} P^{-}}{\gamma+R_{\mathrm{bu}}} & \Rightarrow \frac{R_{\mathrm{a}} \gamma}{H^{2}}+\frac{R_{\mathrm{a}} R_{\mathrm{bu}}}{H^{2}}>R_{\mathrm{bu}} P^{-} \\
& \Rightarrow P^{-}\left(\frac{R_{\mathrm{a}} H^{2}}{H^{2}}-R_{\mathrm{bu}}\right)+\frac{R_{\mathrm{a}} R_{\mathrm{bu}}}{H^{2}}>0 \\
& \Rightarrow P^{-}\left(R_{\mathrm{a}}-R_{\mathrm{bu}}\right)+\frac{R_{\mathrm{a}} R_{\mathrm{bu}}}{H^{2}}>0
\end{aligned}
$$


which is correct if $R_{\mathrm{a}}>R_{\mathrm{bu}}$. Note that if $R_{\mathrm{f}}$ had been used instead of $R_{\mathrm{a}}$, a similar expression is obtained

$$
P^{-}\left(R_{\mathrm{f}}-R_{\mathrm{bu}}\right)+\frac{R_{\mathrm{f}} R_{\mathrm{bu}}}{H^{2}} \approx P^{-}\left(R_{\mathrm{f}}-R_{\mathrm{bu}}\right)<0
$$

which is correct if $R_{\mathrm{f}} \ll R_{\mathrm{bu}}<1$. These results lead to following set of inequalities

$$
P_{\mathrm{af}}^{+}>P_{\mathrm{bu}}^{+}>P_{\mathrm{f}}^{+}
$$

It is also important to show that $P_{\mathrm{af}}^{+}>P_{\mathrm{abu}}^{+}>P_{\mathrm{bu}}^{+}$

$$
\begin{gathered}
P_{\mathrm{af}}^{+} \approx \frac{R_{\mathrm{a}}}{H^{2}} \\
P_{\mathrm{abu}}^{+}=\left(1-\frac{\gamma}{\gamma+R_{\mathrm{bu}}}\right)^{2} P^{-}+\left(\frac{\gamma}{H\left(\gamma+R_{\mathrm{bu}}\right)}\right)^{2} R_{\mathrm{a}}
\end{gathered}
$$

Similar to the derivation of $P_{\mathrm{bu}}^{+}$:

$$
P_{\mathrm{abu}}^{+}=\frac{R_{\mathrm{bu}}^{2} \gamma+\gamma^{2} R_{\mathrm{a}}}{H^{2}\left(\gamma+R_{\mathrm{bu}}\right)^{2}}
$$

Therefore,

$$
\begin{aligned}
P_{\mathrm{af}}^{+}-P_{\mathrm{abu}}^{+} & =\frac{R_{\mathrm{a}}}{H^{2}}-\frac{R_{\mathrm{bu}}^{2} \gamma+\gamma^{2} R_{\mathrm{a}}}{H^{2}\left(\gamma+R_{\mathrm{bu}}\right)^{2}} \\
& =\frac{R_{\mathrm{a}} \gamma^{2}+2 R_{\mathrm{a}} R_{\mathrm{bu}} \gamma+R_{\mathrm{a}} R_{\mathrm{bu}}^{2}-R_{\mathrm{bu}}^{2} \gamma-\gamma^{2} R_{\mathrm{a}}}{H^{2}\left(\gamma+R_{\mathrm{bu}}\right)^{2}} \\
& =\frac{\gamma R_{\mathrm{bu}}\left(2 R_{\mathrm{a}}-R_{\mathrm{bu}}\right)+R_{\mathrm{a}} R_{\mathrm{bu}}^{2}}{H^{2}\left(\gamma+R_{\mathrm{bu}}\right)^{2}}>0
\end{aligned}
$$

Using a similar approach, the following result can be reached

$$
\begin{aligned}
P_{\mathrm{abu}}^{+}-P_{\mathrm{bu}}^{+} & =\frac{R_{\mathrm{bu}}^{2} \gamma+R_{\mathrm{a}} \gamma^{2}-R_{\mathrm{bu}}^{2} \gamma-R_{\mathrm{bu}} \gamma^{2}}{H^{2}\left(\gamma+R_{\mathrm{bu}}\right)^{2}} \\
& =\frac{\gamma^{2}\left(R_{\mathrm{a}}-R_{\mathrm{bu}}\right)}{H^{2}\left(\gamma+R_{\mathrm{bu}}\right)^{2}}>0
\end{aligned}
$$

In conclusion,

$$
P_{\mathrm{af}}^{+}>P_{\mathrm{abu}}^{+}>P_{\mathrm{bu}}^{+}>P_{\mathrm{f}}^{+}
$$

or equivalently

$$
P_{\mathrm{af}}^{+}-P_{\mathrm{f}}^{+}>P_{\mathrm{abu}}^{+}-P_{\mathrm{bu}}^{+}
$$

which confirms that after this update step, the error covariance using the Bump Up $R$ approach $\left(P_{\mathrm{bu}}^{+}\right)$is larger than the ICEKF result $\left(P_{\mathrm{f}}^{+}\right)$and is a better indicator of the actual filter covariance. The main factor that makes $P_{\mathrm{abu}}$ closer to $P_{\mathrm{bu}}$ than $P_{\mathrm{af}}$ is to $P_{\mathrm{f}}$ is the amount by which the noise covariance has been magnified. Since the actual noise covariance, $R_{\mathrm{a}}$, is not known, the bumped-up noise covariance attempts to estimate the value of actual noise covariance.

\section{Measure of improvement}

To measure the improvement obtained by the Bump Up $R$ method, Equation 52 can be normalized. First assume $\alpha R_{\mathrm{bu}}=R_{\mathrm{a}}$ for some $\alpha \geq 1$

$$
\frac{P_{\mathrm{af}}^{+}-P_{\mathrm{abu}}^{+}}{P_{\mathrm{af}}^{+}}=\frac{H^{2}}{R_{\mathrm{a}}} \frac{\gamma R_{\mathrm{bu}}\left(2 R_{\mathrm{a}}-R_{\mathrm{bu}}\right)+R_{\mathrm{a}} R_{\mathrm{bu}}^{2}}{H^{2}\left(\gamma+R_{\mathrm{bu}}\right)^{2}}
$$

Substitute $R_{\mathrm{bu}}=\frac{R_{\mathrm{a}}}{\alpha}$, and Eq. 55 becomes

$$
\frac{P_{\mathrm{af}}^{+}-P_{\mathrm{abu}}^{+}}{P_{\mathrm{af}}^{+}}=1-\frac{R_{\mathrm{a}} \gamma+\alpha^{2} \gamma^{2}}{\left(\alpha \gamma+R_{\mathrm{a}}\right)^{2}}
$$




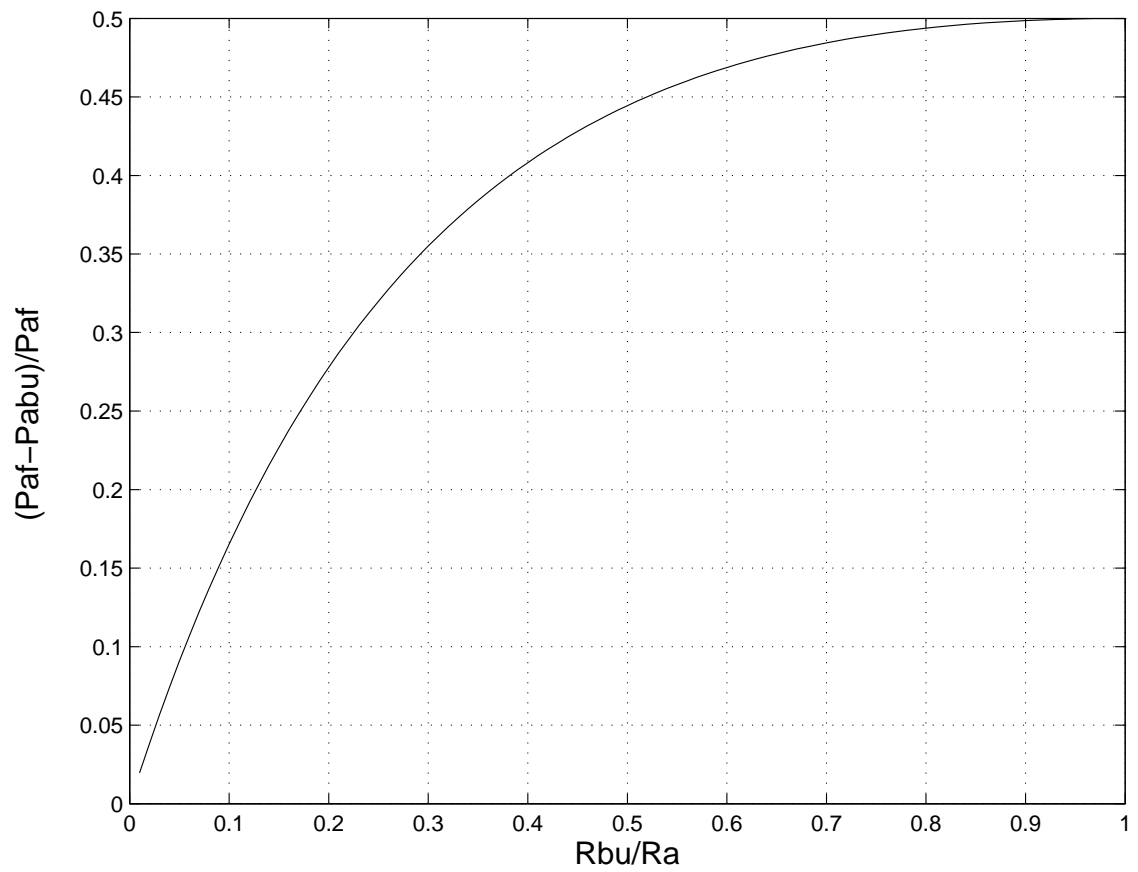

Figure 3. $\frac{P_{\mathrm{af}}^{+}-P_{\mathrm{abu}}^{+}}{P_{\mathrm{af}}^{+}}$as a function of $\frac{R_{\mathrm{bu}}}{R_{\mathrm{a}}}$; When $\frac{R_{\mathrm{bu}}}{R_{\mathrm{a}}}=1$, then $P_{\mathrm{best}}^{+}=P_{\mathrm{abu}}^{+}$and the difference is largest. On the other hand when $R_{\mathrm{bu}} \approx R_{\mathrm{f}}$ then $R_{\mathrm{bu}} \ll R_{\mathrm{a}}$ and the $P_{\mathrm{af}}^{+} \approx P_{\mathrm{abu}}^{+}$. Graph shows a certain difference, which is due the approximation used for calculating $P_{\text {af }}^{+}$

Figure 3 shows the normalized difference between the actual covariances, $P_{\text {abu }}$ and $P_{\text {af }}$, with $\gamma=1$ and $R_{\mathrm{a}}=1$. The value of the normalized difference decreases with increased $\alpha$, which means that as $R_{\mathrm{bu}}$ decreases, $P_{\text {abu }}$ gets closer to $P_{\text {af }}$, which agrees with the analytic derivation. Then, for $\alpha=1, P_{\text {abu }}=P_{\text {best }}$ and the difference is the largest (in this specific case the difference is 50\%). The strength of the SKF approach is that it calculates the best possible value for bumping up the measurement noise covariance at every step.

\section{Simulation}

A simulation was conducted to validate approximations made when obtaining the analytic results and verify that the Bump Up $R$ method performs better than ICEKF. The analysis computed the transient behavior of the error covariance using various filters that were based on different assumptions about the measurement noise covariances $R$. The measurement covariance matrices used in this study are

- The actual value $R_{\mathrm{a}}$

- What is assumed in the ICEKF $R_{\mathrm{f}}$, which is lower than actual $R_{\mathrm{a}}$

- Increased (Bump Up R) value $R_{\mathrm{bu}}$, which takes on values between the actual $R_{f}$ and the filter $R_{f}$

Several different cases were examined:

1. The actual error covariance matrix $P_{\mathrm{a}}$ is computed using $R_{\mathrm{a}}$, but when the gain is calculated with $R_{\mathrm{f}}$ $\left(R_{\mathrm{bu}}\right)$ it is called $P_{\mathrm{af}}\left(P_{\mathrm{abu}}\right)$.

2. The error covariance matrix $P_{\mathrm{f}}$ is analyzed using $R_{\mathrm{f}}$ with the gain based on $R_{\mathrm{f}}$. The same relationship holds for $P_{\mathrm{bu}}$ and $R_{\mathrm{bu}}$.

3. $P_{\text {best }}$ corresponds to the optimal result with the filter designed and analyzed using $R_{\mathrm{a}}$.

The results obtained from comparing these cases are shown in Figs. 4 . The results confirm the analytical comparisons for a wide range of possible $R_{\mathrm{f}}$ and $P^{-}$values. They also show that $P_{\text {abu }}$ is much closer to $P_{\text {best }}$ than $P_{\text {af }}$, indicating that $P_{\mathrm{abu}}$ is a much better predictor of the best possible filter performance. A similar result is expected for the SKF. 

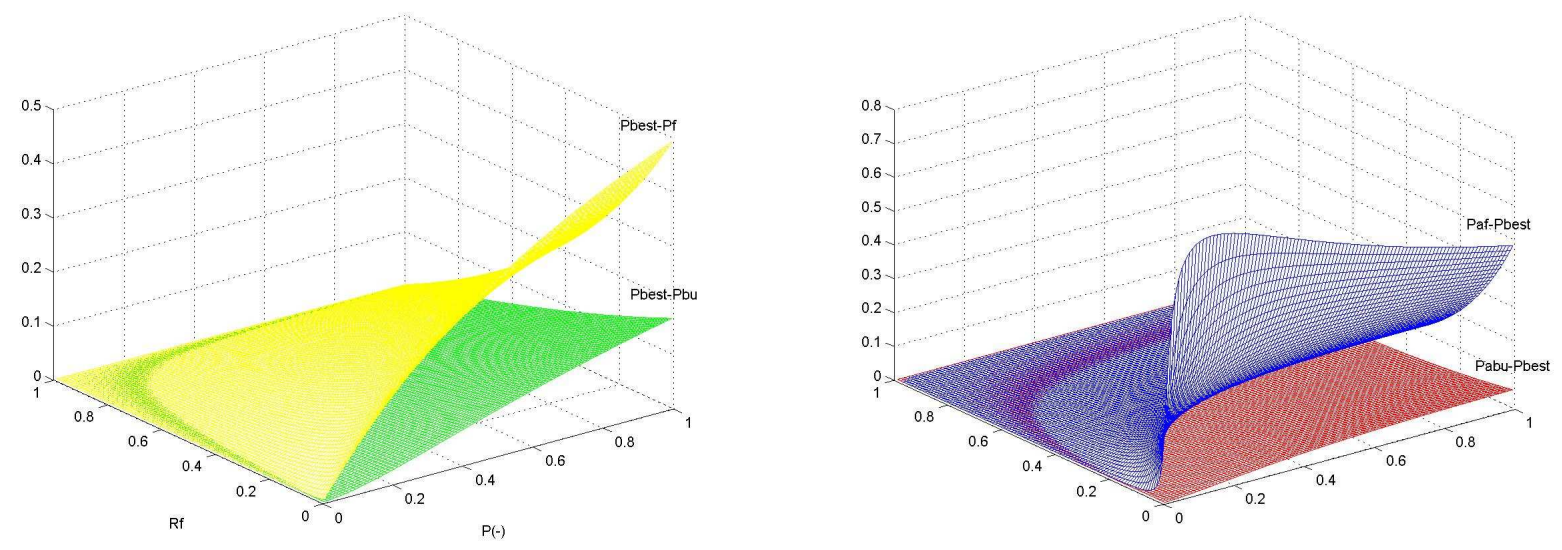

Figure 4. Error Covariance Comparison. This figure actually shows what this section has proven: $P_{\text {af }}>P_{\text {abu }}>$ $P_{\text {best }}>P_{\mathrm{bu}}>P_{\mathrm{f}}$. The bump-up terms in Bump Up R/SK filters are bringing the $P_{\mathrm{af}}$ and $P_{\mathrm{f}}$ to new values $P_{\text {abu }}$ and $P_{\mathrm{bu}}$ which are closer to the best possible covariance value $P_{\mathrm{best}}$

\section{Application of SKF to Hierarchic Architectures}

Previous work has shown the relative merits of centralized and decentralized navigation architectures.14 The principal disadvantages of centralized architectures are high computational load and susceptibility to single-point failure. In a centralized architecture, the primary computational burden is placed on a single spacecraft, severely limiting the size of a formation. Likewise, routing all communication and computation through a single spacecraft creates the potential for a single point failure for the entire formation, should that spacecraft malfunction. Decentralized architectures can avoid both problems by distributing computational load across the formation, with very little performance loss relative the centralized architecture $\frac{14}{14}$ Section III showed that of the decentralized estimators considered, the Schmidt Kalman Filter provides the best performance. This performance advantage is derived from the way the SKF shares and incorporates new information into the estimate. The approach used by the SKF to share specific information between satellites can be applied to other filters, including those using more centralized architectures. This is achieved using the Schmidt covariance correction (SCC), which allows the various estimation architectures/algorithms to correctly account for errors in the range measurements without needing to estimate the states of those vehicles. This can greatly improve the performance and adaptability of the estimation approach, both of which are important properties for reconfigurable networks.

Although using decentralized architectures improves fleet scalability and robustness, it adds complexity to communications and information sharing. This puts limits on the size of the fleet running the decentralized filter. We are currently exploring into the possibilities of developing a new type of hierarchic architecture that will incorporate the use of the SCC. This architecture will be different from a traditional hierarchy, because spacecraft will be allowed to communicate with vehicles in their local cluster and with vehicles in other clusters. We will accomplish this without having to increase the estimator size, by using the Schmidt Covariance Correction. The SCC will enable each spacecraft to range off the spacecraft in other clusters (cross-team ranging) without having to estimate their relative states. The SCC essentially provides each spacecraft with a way to receive and correctly implement new information, regardless of where this information is coming from.

\section{Conclusion}

This paper investigates various approaches to design highly distributed estimators for formation flying applications. It presents a detailed investigation of the covariances for the different distributed estimation algorithms, showing that the Schmidt Kalman Filter and Bump $U p R$ approaches are much better predictors of the best possible filter performance. Finally, we indicate that the main concept behind the Schmidt Kalman Filter (called the Schmidt covariance correction) can be used to develop a reduced-order hierarchic 
estimator that offers distributed computation and can improve the scalability limitations of the centralized and decentralized architectures.

\section{Acknowledgements}

Research funded under NASA Space Communications Project Grant \#NAG3-2839. The authors would like to thank Philip Ferguson and Megan Mitchell for their ideas and suggestions.

\section{References}

${ }^{1}$ J. Leitner, F. Bauer, D. Folta, R. Carpenter, M. Moreau, and J. How, "Formation Flight in Space," GPS World, Feb. 2002, pp. 22-31.

${ }^{2}$ E. C. Aldridge, Jr. et al. "Moon, Mars and Beyond. A Journey to Inspire, Innovate, and Discover," Report of the Presidents Commission on Implementation of United States Space Exploration Policy, June 2004.

${ }^{3}$ L. Breger, P. Ferguson, J. P. How, S. Thomas, and M. Campbell, "Distributed Control of Formation Flying Spacecraft Built on OA," Proceedings of the AIAA Guidance, Navigation, and Control Conf., August 2003. (Paper 2003-5366)

${ }^{4}$ F. D. Busse, Precise Formation-State Estimation in Low Earth Orbit using Carrier Differential GPS. Ph.D. thesis, Stanford University, Dept. of Aero/Astro, Nov. 2002.

${ }^{5}$ T. Corazzini, Onboard Pseudolite Augmentation for Spacecraft Formation Flying. Ph.D. Dissertation, Stanford University, Dept. of Aeronautics and Astronautics, Aug. 2000.

${ }^{6}$ C.-W. Park, J. How, and L. Capots, "Sensing Technologies for Formation Flying Spacecraft in LEO Using Inter-Spacecraft Communications System," the Navigation J. of the Institute of Navigation, Vol. 49, No. 1, Spring 2002, pp. 45-60.

${ }^{7}$ J. R. Carpenter, C. Gramling et al, "Relative Navigation of Formation-Flying Satellites," from the proceedings of the International Symposium on Formation Flying, Toulouse France, October 2002.

${ }^{8}$ P. Stadter, R. Heins, et al., "Enabling Distributed Spacecraft Systems with the Crosslink Transceiver," AIAA Space Conference and Exposition, Aug. 2001. Paper 2001-4670.

${ }^{9}$ G. Purcell, D.Kuang, S. Lichten, S.C. Wu and L. Young, "Autonomous Formation Flyer (AFF) Sensor Technology Development," TMO Progress Report 42-134, August 1998.

${ }^{10}$ C. Park and J. P. How, "Precise Relative Navigation using Augmented CDGPS," ION-GPS Conference, Sept. 2001.

${ }^{11} \mathrm{P}$. Ferguson, T. Yang, M. Tillerson and J. How, "New Formation Flying Testbed for Analyzing Distributed Estimation and Control Architectures." Presented at the AIAA GNC, Monterey, CA, August 2002.

${ }^{12}$ P. Ferguson and J. P. How, "Decentralized Estimation Algorithms for Formation Flying Spacecraft," Proceedings of the AIAA Guidance, Navigation, and Control Conf., August 2003. (Paper 2003-5442)

${ }^{13}$ C. W. Park, Precise Relative Navigation using Augmented CDGPS. Ph.D. thesis, Stanford University, Dept. of Mech. Eng., June 2001.

${ }^{14}$ P. Ferguson, Distributed Estimation and Control Technologies for Formation Flying Spacecraft. S.M. thesis, Massachusetts Institute of Technology, Dept. of Aero/Astro, Jan. 2003.

${ }^{15}$ R. Brown, P. Hwang, Introduction to Random Signals and Applied Kalman Filtering. Third Ed., pp. 366-7, John Wiley \& Sons, 1997. 